\title{
SESQUITERPENES FROM LEAVES OF CRYPTOMERIA JAPONICA
}

\author{
Wen-Chiung Su, Jim-Min FAng and Yu-Shia Cheng* \\ Department of Chemistry, National Taiwan University, Taipei, Tajwan 106, Republic of China
}

(Received in revised form 21 November 1994)

Key Word Index — Cryptomeria japonica; Taxodiaceae; leaves; sesquiterpenes.

\begin{abstract}
Twenty-seven sesquiterpenes were isolated from leaves of Cryptomeria japonica. The new compounds included elem-1-en-4,11-diol, 11 -acetoxyeudesman-4 $\alpha$-ol, eudesmane-5 $\alpha, 11$-diol, 3-eudesmene-1 $\beta, 11$-diol, $1 \beta$-acetoxy3-eudesmen-11-ol, 4-eudesmene-1 $\beta, 11$-diol, $1 \beta$-acetoxy-4-eudesmen-11-ol, 7-epi-y-eudesmol, 7-epi-4-eudesmene$1 \beta, 11$-diol, $1 \beta$-acetoxy-4(15)-eudesmen-11-ol. Their structures were determined by chemical and spectral methods.
\end{abstract}

\section{INTRODUCTION}

The Japanese cedar, Cryptomeria japonica D. Don., is a widely distributed conifer called 'sugi' in Japanese. We recently reported the isolation and structural determination of chamaecydin triterpene [1], abietane, kaurane and labdane diterpenes $[2,3]$ from the ethyl acetatesoluble part of the leaves of $C$. japonica. As a continuation of this study, we describe herein, 27 constituents of sesquiterpenes including 10 novel compounds $5,8,9,11$, 12, 14-17, and 23.

\section{RESULTS AND DISCUSSION}

The leaves of $C$. japonica were extracted with acetone. The ethyl acetate-soluble portion of the extract was subjected to chromatography to give sesquiterpenes 1-27. The known compounds epitodomatuic acid (1) [4], epijuvabione (2) [4], 11-hydroxy-4,5-secoeudesmane-4,5dione (3) [5], elemol (4) [6], cryptomeridiol (6) [7], 4epicryptomeridiol (7) [8], $\alpha$-eudesmol (10) $[9,10], \gamma$ eudesmol (13) [9], 6-eudesmene-1 $\beta, 4 \beta$-diol (18) [11], oplodiol (19) $[10,12], \beta$-eudesmol (20) $[13], 4(15)$ eudesmene-1 $\beta, 11$-diol (21) [14], 4(15)-eudesmene-1 $\beta, 6 \alpha$ $\operatorname{diol}(22)[15,16], \alpha$-cadinol (24) [17], T-cadinol (25) [18], oplopanone (26) [19] and cedrol (27) [20] were identified by comparison of their physical and spectral data $(\mathrm{mp}$, $[\alpha]$, mass, IR, ${ }^{1} \mathrm{H}$ and ${ }^{13} \mathrm{CNMR}$ ) with literature.

The molecular formula, $\mathrm{C}_{15} \mathrm{H}_{28} \mathrm{O}_{2}$, of 5 was inferred from its exact mass 240.209 . The ${ }^{13} \mathrm{CNMR}$ spectrum showed signals for a terminal double bond at $\delta 153.3(d)$ and $109.5(t)$. The signals for two isopropanol moieties appeared at $\delta 1.17(\mathrm{Me}), 1.18(3 \mathrm{Me}), 72.7(\mathrm{~s})$ and $75.4(s)$. The proton resonance at $\delta 1.57$ was assigned to $\mathrm{H}-5$ by means of $\mathrm{C}-\mathrm{H}$ COSY and HMBC. As $\mathrm{H}-5$ appeared as a double of doublet $(J=12,3 \mathrm{~Hz})$, it was in the axial

*Author to whom correspondence should be addressed. orientation. Compound 5 had chemical shifts for C-7 and $C-10$ at $\delta 49.5(d)$ and $40.3(s)$, close to the values for those signals in elemol. The structure of 5 was determined to be elem-1-en-4,11-diol and its $(5 R, 7 R, 10 S)$-configuration was tentatively assigned by analogy to that of elemol. Compound 5 was unstable in $\mathrm{CDCl}_{3}$ solution. A product 5a, (1S)-elemane-1,4,11-triol, was obtained presumably by the acid-catalysed hydration of 5 . The $S$-configuration was determined by Horeau's method [21]. Compound 5 can be also regarded as a hydration derivative of elemol (4).

From spectral analyses, $8\left(\mathrm{C}_{17} \mathrm{H}_{30} \mathrm{O}_{3}\right)$ was readily determined to be 11-acetoxyeudesman- $4 \alpha-$ ol. It showed the IR absorption at $1723 \mathrm{~cm}^{-1}$ and carbon resonances at $\delta 22.4$ and 170.5 for the acetoxy group. The structure of 8 was confirmed as it was saponified to yield cryptomeridiol (6). Two C-11 methyl groups in 6 appearing at $\delta 1.16$ were deshielded to $\delta 1.40$ and 1.43 in 8 as the C-11 hydroxyl group was converted to the acetoxy group.

An eudesmanediol (9) $\left(\mathrm{C}_{15} \mathrm{H}_{28} \mathrm{O}_{2}\right)$ exhibited the parent peak in the mass spectrum at $m / z$ 240.209. The $\mathrm{C}-\mathrm{H}$ COSY and HMBC experiments led to the assignment of 9 as eudesmane-5 $\alpha, 11$-diol. Irradiation of Me-10 (at $\delta 0.93$ ) caused $7 \%$ NOE of Me-4 (at $\delta 0.81$ ) and $10 \%$ NOE of $\mathrm{H}$ $6 \beta$ (at $\delta 1.28$ ). The signal of $H-6 \alpha$ appeared at a relatively low field $\delta 1.72$ presumably due to the deshielding effect of the $5 \alpha$-hydroxyl group. The large coupling constant $12.5 \mathrm{~Hz}$ between $\mathrm{H}-6 \beta$ and $\mathrm{H}-7$ was in agreement with their axial positions.

Based on the spectral analyses, two isomers $\left(\mathrm{C}_{15} \mathrm{H}_{26} \mathrm{O}_{2}\right) 11$ and 14 , were assigned as 3-eudesmene$1 \beta, 11$-diol and 4-eudesmene-1 $\beta, 11$-diol, respectively. Two olefinic carbons in 11 occurred at $\delta 119.5(d)$ and $135.4(s)$, whereas those in 14 appeared at $\delta 123.8(s)$ and $133.6(s)$. The H-1 in 11 was axially oriented to exhibit its resonance as a double of doublet (at $\delta 3.50$ ) with 10 and $6.5 \mathrm{~Hz}$ coupling constants. The resonance of H-1 in 14 also showed a similar pattern $(d d, J=9,7 \mathrm{~Hz})$. An allylic 
<smiles>[R]C([R])([R])C1=CCC([C@H](C)CC(=O)CC(C)C)CC1</smiles>

$1 \mathbf{R}=\mathbf{H}$

$2 \mathrm{R}=\mathrm{CH}_{3}$<smiles>C=C[C@]1(C)CCC(C(C)(C)O)C[C@H]1C(C)(C)O</smiles><smiles>C[C@H]1CCC[C@]2(C)CCC(C(C)(C)O)C[C@]12O</smiles>

9<smiles>[R]C1CCC(C)=C2C[C@H](C(C)(C)O)CC[C@@]21C</smiles>

$16 R=H$

$17 \mathrm{R}=\mathrm{OH}$<smiles>[R4]C1[C@@H]2[C@H]([R])CCC(=C)[C@@]2(C)CC[C@H]1C([R])(C)C</smiles>

$\begin{array}{llll} & \mathbf{R}^{1} & \mathbf{R}^{2} & \mathbf{R}^{3} \\ 20 & \mathbf{H} & \mathbf{H} & \text { OH } \\ \mathbf{2 1} & \text { OH } & \mathbf{H} & \text { OH } \\ \mathbf{2 2} & \text { OH } & \text { OH } & \mathbf{H} \\ 23 & \text { OAc } & \mathbf{H} & \text { OH }\end{array}$<smiles>CC(=O)CCC[C@]1(C)CCC(C(C)(C)O)CC1=O</smiles>

3<smiles>C[C@H](O)[C@@]1(C)CCC(C(C)(C)O)C[C@H]1C(C)(C)O</smiles><smiles>[R]C1CC=C(C)C2CC(C(C)(C)O)CC[C@]12C</smiles>

$10 R=H$

$11 \mathrm{R}=\mathrm{OH}$

$12 \mathrm{R}=\mathrm{OAc}$<smiles>CC(C)C1=C[C@@H]2[C@@](C)(O)CC[C@H](O)[C@@]2(C)CC1</smiles>

18<smiles>CC(C)C1=CC[C@]2(C)C(C1)[C@@](C)(O)CC[C@@H]2O</smiles>

19<smiles></smiles>

26<smiles>C=C(C)C1C[C@H](C(C)(C)O)CC[C@]1(C)C(C)C</smiles><smiles>[R]C(C)(C)C1CC[C@]2(C)C(C1)C([R])([R])CCCC2(C)C</smiles>
$\begin{array}{lll}\mathbf{R}^{1} & \mathbf{R}^{2} & \mathbf{R}^{3}\end{array}$
$6 \mathrm{CH}_{3}$ OH $\mathrm{OH}$
$\begin{array}{llll}7 \mathrm{OH} & \mathrm{CH}_{3} & \mathrm{OH}\end{array}$
$8 \mathrm{CH}_{3} \mathrm{OH} \mathrm{OAc}$

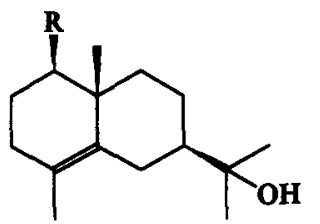

$13 R=H$

$14 \mathrm{R}=\mathrm{OH}$

$15 R=O A c$<smiles>[R]CC(C[R]([H])([H])O)[C@H]1CCC([R])([Y1])[C@H]2CCC(C)=C[C@H]12</smiles>

proton $\mathrm{H}-5$ appearing at $\delta 1.66(d d, J=9,3 \mathrm{~Hz})$ also conformed to the trans-fused configuration of 11 . The C-7 resonances of 11 and 14 occurred at $\delta 49.2$ and 49.7 close to the value of C-7 ( $\delta 50.0)$ in $\alpha$-eudesmol (10).

Compounds 12 and 15 are $1 \beta$-acetoxy-3-eudesmen-11ol and $1 \beta$-acetoxy-4-eudesmen-11-ol, the acetates of 11 and 14, respectively. Due to the inductive effect of acetoxy groups, the $\mathrm{H}-1$ resonances in $\mathbf{1 2}$ and 15 occurred at low fields $\delta 4.74(d d, J=9.5,6.5 \mathrm{~Hz})$ and $4.67(d d, J=8,8 \mathrm{~Hz})$.
Saponification of 12 and 15 gave, respectively, the corresponding diol 11 and 14.

Compounds $16\left(\mathrm{C}_{15} \mathrm{H}_{26} \mathrm{O}\right)$ and $17\left(\mathrm{C}_{15} \mathrm{H}_{26} \mathrm{O}_{2}\right)$ were determined to be 7-epi- $\gamma$-eudesmol and 7-epi-4eudesmene-1 $\beta, 11$-diol, respectively. The coupling constants between the $\mathrm{C}-7$ and $\mathrm{C}-6$ protons were small $(2-3.5 \mathrm{~Hz})$ as the $C-7$ protons were on equatorial positions. The C-7 signals in 16 and 17 appeared at $\delta 44.1$, whereas those signals in their 7-epimers 13 and 14 
appeared at lower fields ( $\Delta \delta=5 \mathrm{ppm})$ [22]. In contrast, the $\mathrm{C}-11$ signals of the isopropanol moieties in $\mathbf{1 6}$ and 17 occurred at lower fields than those in $\mathbf{1 3}$ and 14.

Compound 23 showed IR absorption at 3453 (broad) and $1711 \mathrm{~cm}^{-1}$ attributable to hydroxyl and acetyl groups. The exact mass at $m / z 280.202$ indicated the molecular formula $\mathrm{C}_{17} \mathrm{H}_{28} \mathrm{O}_{3}$ and an intense signal at $m / z 202$ was attributable to the fragment derived by elimination of water and acetic acid $\left[\mathrm{M}-\mathrm{H}_{2} \mathrm{O}\right.$ $-\mathrm{HOAc}]^{+}$.By analysis of the ${ }^{1} \mathrm{H}$ and ${ }^{13} \mathrm{C}$ NMR spectra, the structure of 23 was determined to be $1 \beta$-acetoxy- 4 (15)-eudesmen-11-ol. To confirm this structural assignment, 23 was saponified to give the eudesmenediol 21.

In summary, a series of known and new sesquiterpenes were isolated from the leaves of $C$. japonica. The structures of new sesquiterpenes were determined by analyses of their spectra. The acetates 8, 12, 15 and 23 were correlated with their corresponding alcohols by saponification.

\section{EXPERIMENTAL}

General. Merck silica gel $60 \mathrm{~F}$ sheets were used for analyt. TLC. HPLC was carried out on a Hibar Lichrosorb Si $60(7 \mu \mathrm{m}$ or $10 \mu \mathrm{m})$ column $(25 \mathrm{~cm} \times 1 \mathrm{~cm})$.

Plant material. The plant used in this study is introduced from Japan and cultivated in suburban Taipei. A voucher specimen has been deposited in our laboratory. The leaves $(1.4 \mathrm{~kg})$ of $C$. japonica $\mathrm{D}$. Don. were exhaustively extracted with $\mathrm{Me}_{2} \mathrm{CO}$. The $\mathrm{Me}_{2} \mathrm{CO}$ extract was passed through a pad of charcoal, concd and re-extracted with EtOAc. The EtOAc-soluble portion $(45 \mathrm{~g})$ was chromatographed on a silica gel column by elution with gradient of hexane and EtOAc. The appropriate frs were combined and purified by HPLC to give $16(20 \mathrm{mg}), 2$ (16 mg), 24 (12 mg), 25 (8 mg), 27 (22 mg), 23 (27 mg), 4 (12 mg), $10(8 \mathrm{mg}), 20$ (13 mg), 8 (27 mg), 15 (10 mg), 12 (11 mg), 13 (15 mg), 26 (20 mg), 9 (21 mg), 1 (15 mg), 19 (8 mg), 17 (3 mg), 22 (12 mg), 11 (14 mg), 14 (50 mg), 3 (3 mg), 21 (15 mg), $18(5 \mathrm{mg}), 7(35 \mathrm{mg}), 5(5 \mathrm{mg})$ and 6 $(25 \mathrm{mg})$, in order of increasing polarity.

Epitodomatuic acid (1). Oil, $[\alpha]_{\mathrm{D}}^{20}+71^{\circ}\left(\mathrm{CHCl}_{3} ; c 1.5\right)$, lit. [4], Oil, $[\alpha]_{\mathrm{D}}^{25}+71.2^{\circ}\left(\mathrm{CHCl}_{3} ; c 1.07\right)$.

Epijuvabione (2). Oil, $[\alpha]_{\mathrm{D}}^{25}+60^{\circ}\left(\mathrm{CHCl}_{3} ; c 1.6\right)$, lit. [4], Oil, $[\alpha]_{\mathrm{D}}^{25}+60^{\circ}\left(\mathrm{CHCl}_{3} ; c 1.18\right)$.

11-Hydroxy-4,5-secoeudesmane-4,5-dione (3). Oil, $[\alpha]_{\mathrm{D}}^{15}$ $+50^{\circ}\left(\mathrm{CHCl}_{3} ; c 0.3\right)$, lit. [5], Oil, $[\alpha]_{\mathrm{D}}^{24}+46^{\circ}\left(\mathrm{CHCl}_{3}\right.$; c 0.39). ${ }^{13} \mathrm{CNMR}\left(\mathrm{CDCl}_{3}, 75 \mathrm{MHz}\right): \delta 18.3$ (C-2), 21.9 (C8), 23.1 (C-14), 27.2 (C-12), 27.4 (C-13), 29.9 (C-15), 36.3 (C-9), 37.3(C-1), 39.8 (C-6), 44.3(C-3), 47.1 (C-10), 49.6(C7), 72.0 (C-11), 209.1 (C-4), 215.8 (C-5).

Elemol (4). Oil, $[\alpha]_{\mathrm{D}}^{25}-6^{\circ}\left(\mathrm{CHCl}_{3} ; \mathrm{c} \mathrm{1.8}\right)$, lit. [6], mp $52-53^{\circ} ;[\alpha]_{\mathrm{D}}-5.82^{\circ}\left(\mathrm{CHCl}_{3} ; c 3.4\right) .{ }^{13} \mathrm{CNMR}\left(\mathrm{CDCl}_{3}\right.$, $75 \mathrm{MHz}): \delta 16.6$ (C-14), 22.5 (C-8), 24.7 (C-15), 27.1 (C-12, 13), 28.5 (C-6), 39.7 (C-10), 39.9 (C-9), 49.3 (C-7), 52.7 (C5), 72.7 (C-11), 109.9 (C-2), 112.0 (C-3), 147.9 (C-4), 150.2 (C-1).

Elem-1-en-4,11-diol (5). Oil, $[\alpha]_{\mathrm{D}}^{15}-14^{\circ}\left(\mathrm{CHCl}_{3} ; c 0.5\right)$. TLC (50\% EtOAc in hexane) $R_{f} 0.44$. IR $v_{\max }^{\text {neat }} \mathrm{cm}^{-1}: 3388$, $3081,1625,914$. EIMS (70 eV) $m / z$ (rel. int.): $240[\mathrm{M}]^{+}$ 
Table $2 .{ }^{13} \mathrm{CNMR}$ spectral data of new compounds (75 $\mathrm{MHz}, \mathrm{CDCl}_{3}$ solution, $\delta$ values in ppm)

\begin{tabular}{|c|c|c|c|c|c|c|c|c|c|c|}
\hline C & 5 & 8 & 9 & 11 & 12 & 14 & 15 & 16 & 17 & 23 \\
\hline 1 & 153.3 & 41.0 & 33.0 & 76.3 & 78.2 & 78.3 & 80.3 & 39.4 & 75.4 & 80.7 \\
\hline 2 & 109.5 & 20.2 & 22.2 & 32.3 & 29.1 & 27.1 & 23.9 & 18.9 & 26.9 & 28.0 \\
\hline 3 & 31.5 & 43.6 & 30.5 & 119.5 & 119.0 & 31.9 & 31.5 & 32.7 & 31.3 & 33.8 \\
\hline 4 & 75.4 & 72.2 & 32.4 & 135.4 & 135.3 & 123.8 & 124.0 & 126.0 & 125.1 & 148.2 \\
\hline 5 & 55.7 & 54.8 & 75.2 & 46.5 & 46.4 & 133.6 & 133.4 & 135.0 & 133.6 & 48.7 \\
\hline 6 & 23.0 & 21.2 & 32.1 & 23.9 & 23.7 & 26.5 & 26.3 & 25.4 & 25.2 & 24.2 \\
\hline 7 & 49.5 & 47.2 & 45.2 & 49.2 & 49.1 & 49.7 & 49.7 & 44.1 & 44.1 & 47.6 \\
\hline 8 & 25.5 & 22.1 & 21.3 & 21.9 & 21.8 & 22.8 & 22.7 & 22.6 & 21.8 & 21.9 \\
\hline 9 & 43.8 & 44.4 & 36.3 & 35.0 & 34.9 & 38.8 & 38.6 & 38.1 & 33.5 & 36.6 \\
\hline 10 & 40.3 & 34.6 & 37.6 & 37.4 & 36.3 & 39.4 & 38.4 & 34.4 & 39.5 & 39.1 \\
\hline 11 & 72.7 & 85.1 & 72.5 & 72.9 & 72.8 & 72.7 & 72.6 & 74.6 & 74.1 & 72.7 \\
\hline 12 & 27.5 & 23.5 & 26.9 & 26.7 & 26.7 & 26.7 & 26.7 & 27.8 & 27.4 & 27.0 \\
\hline 13 & 28.2 & 23.7 & 27.0 & 27.6 & 27.6 & 27.1 & 27.2 & 29.8 & 29.4 & 27.2 \\
\hline 14 & 17.1 & 18.6 & 22.7 & 9.5 & 10.6 & 17.3 & 18.4 & 25.9 & 19.2 & 11.2 \\
\hline 15 & 26.6 & 22.5 & 14.9 & 20.9 & 21.2 & 18.9 & 18.9 & 19.6 & 19.3 & 107.2 \\
\hline \multirow[t]{2}{*}{ OAc } & & 170.5 & & & 171.0 & & 171.0 & & & 170.9 \\
\hline & & 22.4 & & & 20.7 & & 21.3 & & & 21.2 \\
\hline
\end{tabular}

(10), 239 (95), 221 (7), 154 (20), 134 (55), 98 (65), 43 (100). $\mathrm{HRMS}$ for $\mathrm{C}_{15} \mathrm{H}_{28} \mathrm{O}_{2}$ requires 240.2091 ; found 240.2085 .

(1S)-Elemane-1,4,11-triol (5a). This was obtained when 5 stood in $\mathrm{CDCl}_{3}$ soln for 2 weeks. White crystals from $\mathrm{CHCl}_{3}$-hexane $(1: 1)$, mp 93-94, $[\alpha]_{\mathrm{D}}^{30}-3.3^{\circ}\left(\mathrm{CHCl}_{3}\right.$; c 0.3). TLC (30\% EtOAc in hexane) $R_{f}$ 0.47. IR $v_{\max }^{\mathrm{KBr}} \mathrm{cm}^{-1}: 3435 .{ }^{1} \mathrm{H}$ NMR $\left(\mathrm{CDCl}_{3}, 300 \mathrm{MHz}\right): \delta 0.82(s$, $\mathrm{H}-14), 1.05$ ( $s, \mathrm{H}-15), 1.07(d, J=6.5 \mathrm{~Hz}, \mathrm{H}-2), 1.18(s, \mathrm{H}-$ 3), $1.20(s, \mathrm{H}-12), 1.21(s, \mathrm{H}-13), 1.50(d d, J=3,12 \mathrm{~Hz}, \mathrm{H}-$ 5), $3.45(q, J=6.5 \mathrm{~Hz}, \mathrm{H}-1) .{ }^{13} \mathrm{CNMR}\left(\mathrm{CDCl}_{3}, 75 \mathrm{MHz}\right)$ : $\delta 13.5$ (C-2), 14.0 (C-14), 21.8 (C-6), 21.9 (C-8), 24.0 (C-15), 27.1 (C-12), 27.8 (C-13), 30.4 (C-3), 36.0 (C-9), 43.5 (C-10), 49.5 (C-7), 58.1 (C-5), 72.9 (C-11), 78.8 (C-4), 81.6 (C-1). EIMS $(70 \mathrm{eV}) m / z$ (rel. int.): $225\left[\mathrm{M}-\mathrm{H}_{2} \mathrm{O}-\mathrm{Me}\right]^{+}(42)$, 207 (3), 178 (70), 163 (50), 135 (100), 121 (18), 95 (15). HRMS for $\left[\mathrm{C}_{15} \mathrm{H}_{30} \mathrm{O}_{3}-\mathrm{H}_{2} \mathrm{O}-\mathrm{Me}\right]$ requires 225.1856; found 225.1855. A sample of 5a was treated with $( \pm)$-2-phenylbutanoic anhydride in pyridine at $25^{\circ}$ for $1 \mathrm{hr}$. The recovered 2-phenylbutanoic acid after work up showed levorotation, 5a was thus assigned to have (1S)-configuration [21].

Cryptomeridiol (6). Mp 136-137 $.[\alpha]_{\mathrm{D}}^{25}-33^{\circ}\left(\mathrm{CHCl}_{3}\right.$; $c 1.5)$, lit. [7], $m p 137.5^{\circ} ;[\alpha]_{\mathrm{D}}^{20}-21.7^{\circ}\left(\mathrm{CHCl}_{3} ; c 2.5\right)$.

4-Epicryptomeridiol (7). Mp 81-82. $\quad[\alpha]_{\mathrm{D}}^{25}+4^{\circ}$ $\left(\mathrm{CHCl}_{3} ; c 2.5\right)$, lit. [8], mp 81-82 $;[\alpha]_{\mathrm{D}}+3.8^{\circ}\left(\mathrm{CHCl}_{3}\right.$; c 0.22).

11-Acetoxyeudesman- $4 \alpha$-ol (8). Oil, $[\alpha]_{\mathrm{D}}^{25}-13^{\circ}$ $\left(\mathrm{CHCl}_{3} ; c 2.7\right)$. TLC $\left(9 \%\right.$ EtOAc in $\left.\mathrm{CH}_{2} \mathrm{Cl}_{2}\right) R_{f}$ 0.33. IR $v_{\max }^{\text {neat }} \mathrm{cm}^{-1}: 3423,1723$. EIMS (70 eV) $m / z$ (rel. int.): 282 $[\mathrm{M}]^{+}(2), 281(\mathrm{M}-\mathrm{H})^{+}(7), 222[\mathrm{M}-\mathrm{MeCOOH}]^{+}(35)$, 204 (52), 189 (21), 161 (20), 149 (35), 109 (25), 81 (30), 43 (100). HRMS for $\mathrm{C}_{17} \mathrm{H}_{30} \mathrm{O}_{3}$ requires 282.2196; found 282.2175 .

Saponification of 8 . A soln of $8(20 \mathrm{mg})$ in EtOH $(5 \mathrm{ml})$ was treated with $10 \% \mathrm{KOH}$ in EtOH $(2 \mathrm{ml})$ at $25^{\circ}$ for $16 \mathrm{hr}$. The mixt. was extracted with $\mathrm{Et}_{2} \mathrm{O}$ and sepd by
HPLC (30\% EtOAc in hexane) to give $8(10 \mathrm{mg})$ and 6 (8 mg).

Eudesmane-5 $\alpha$-11-diol (9). Solid, mp 66-67.$[\alpha]_{\mathrm{D}}^{25}$ $+38^{\circ}\left(\mathrm{CHCl}_{3} ; c 2.1\right)$. TLC (15\% EtOAc in $\left.\mathrm{CHCl}_{3}\right) R_{f}$ 0.32. IR $v_{\max }^{\mathrm{KBr}} \mathrm{cm}^{-1}: 3465$. EIMS $(70 \mathrm{eV}) \mathrm{m} / z$ (rel. int.): 240 $[\mathrm{M}]^{+}(13), 222(12), 207$ (22), 181 (20), 164 (18), 149 (42), 126 (100), 112 (82). HRMS for $\mathrm{C}_{15} \mathrm{H}_{28} \mathrm{O}_{2}$ requires 240.2090; found 240.2092.

$\alpha$-Eudesmol (10). Oil, $[\alpha]_{\mathrm{D}}^{25}+28^{\circ}\left(\mathrm{CHCl}_{3} ; c 0.8\right)$, lit. $[9], \mathrm{mp} \mathrm{75^{ \circ }} ;[\alpha]_{\mathrm{D}}+28.6^{\circ}\left(\mathrm{CHCl}_{3} ; \mathrm{c} 1.86\right)$.

3-Eudesmene-1ß-11-diol (11). Needles from $\mathrm{CHCl}_{3}-$ hexane $(7: 3), \mathrm{mp} 144-145^{\circ} .[\alpha]_{\mathrm{D}}^{25}-4^{\circ}\left(\mathrm{CHCl}_{3} ; c 1.4\right)$, TLC (33\% EtOAc in $\left.\mathrm{CH}_{2} \mathrm{Cl}_{2}\right) R_{f}$ 0.52. IR $v_{\max }^{\mathrm{KBr}} \mathrm{cm}^{-1}$ : 3333. EIMS (70 eV) $m / z$ (rek. int.): $238\left[\mathrm{M}^{+}\right.$(7), 220 (15), 202 (5), 177 (15), 121 (25), 93 (38), 59 (100). HRMS for $\mathrm{C}_{15} \mathrm{H}_{26} \mathrm{O}_{2}$ requires 238.1934; found 238.1939.

$1 \beta$-Acetoxy-3-eudesmen-11-ol (12). Oil, $[\alpha]_{\mathrm{D}}^{28}+15.5^{\circ}$ $\left(\mathrm{CHCl}_{3} ; c 1.1\right)$. TLC (20\% EtOAc in hexane) $R_{f} 0.4$. IR $v_{\max }^{\text {neat }} \mathrm{cm}^{-1}: 3443,1730$. EIMS (70 eV) $\mathrm{m} / z$ (rel. int.): 280 $[\mathrm{M}]^{+}(1), 262(3), 235$ (13), 220 (20), 203 (85), $187(30), 159$ (35), 145 (50), 43 (100). HRMS for $\mathrm{C}_{17} \mathrm{H}_{28} \mathrm{O}_{3}$ requires 280.2039 ; found 280.2058 . Saponification of $12(10 \mathrm{mg})$ by a procedure similar to that for 8 gave $11(8 \mathrm{mg})$.

$\gamma$-Eudesmol (13). Mp 72-73 $.[\alpha]_{\mathbf{D}}^{25}+21^{\circ}\left(\mathrm{CHCl}_{3}\right.$; c 1.5), lit. [9], mp 73-74 $;[\alpha]_{\mathrm{D}}+18.7^{\circ}\left(\mathrm{CHCl}_{3} ; c 0.7\right)$.

4-Eudesmene-1 $\beta, 11-$ diol (14). Crystals from $\mathrm{CH}_{2} \mathrm{Cl}_{2}$-hexane $(6: 4)$, mp 137-138 $. \quad[\alpha]_{\mathrm{D}}^{15}+61^{\circ}$ $\left(\mathrm{CHCl}_{3} ; c 5.0\right)$. TLC $\left(33 \%\right.$ EtOAc in $\left.\mathrm{CH}_{2} \mathrm{Cl}_{2}\right) R_{f} 0.38$. IR $v_{\max }^{\mathrm{KBr}} \mathrm{cm}^{-1}: 3436$. EIMS (70 eV) $m / z$ (rel. int.): 238 [M] $^{+}$ (15), 220 (100), 203 (40), 187 (35), 159 (48), 133 (35), 119 (25). HRMS for $\mathrm{C}_{15} \mathrm{H}_{26} \mathrm{O}_{2}$ requires 238.1934; found 238.1932.

1B-Acetoxy-4-eudesmen-11-ol (15). Oil, $[\alpha]_{\mathrm{D}}^{28}+60^{\circ}$ $\left(\mathrm{CHCl}_{3} ; c 1.0\right)$. TLC (20\% EtOAc in hexane) $R_{f} 0.44$. IR $v_{\max }^{\text {neat }} \mathrm{cm}^{-1}: 3451,1731$. EIMS $(70 \mathrm{eV}) \mathrm{m} / z$ (rel. int.): 280 $[\mathrm{M}]^{+}(8), 262(30), 220(12), 202(75), 187(70), 159$ (100), 
145 (40), 131 (70). HRMS for $\mathrm{C}_{17} \mathrm{H}_{28} \mathrm{O}_{3}$ requires 280.2039; found 280.2065. Saponification of $15(10 \mathrm{mg})$ by a procedure similar to that for 8 gave $14(8 \mathrm{mg})$.

7-Epi- $\gamma$-eudesmol (16). Oil, $[\alpha]_{\mathrm{D}}^{25}-45^{\circ}\left(\mathrm{CHCl}_{3} ; c 2.0\right)$. TLC $\left(50 \% \mathrm{CHCl}_{3}\right.$ in hexane) $R_{f} 0.3$. IR $v_{\max }^{\text {neat }} \mathrm{cm}^{-1}: 3419$. EIMS (70 eV) $m / z$ (rel. int.): $222\left[\right.$ [M] $^{+}$(22), 204 (95), 189 (80), 161 (100), 149 (25), 133 (35), 119 (18). HRMS for $\mathrm{C}_{15} \mathrm{H}_{26} \mathrm{O}$ requires 222.1985; found 222.1988 .

7-Epi-4-eudesmene-1ß,11-diol (17). Oil, $[\alpha]_{\mathrm{D}}^{25}-15^{\circ}$ $\left(\mathrm{CHCl}_{3} ; c 0.2\right)$. TLC (30\% EtOAc in hexane) $R_{f} 0.25$. IR $v_{\max }^{\text {neat }} \mathrm{cm}^{-1}: 3395,1647$. EIMS $(70 \mathrm{eV}) \mathrm{m} / z$ (rel. int.): 238 $[\mathrm{M}]^{+}(4), 220(15), 202(10), 187(11), 159(17), 105(20), 59$ (60), 43 (100). HRMS for $\mathrm{C}_{15} \mathrm{H}_{26} \mathrm{O}_{2}$ requires 238.1934; found 238.1938 .

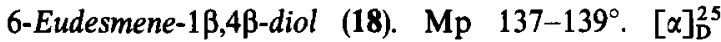
$-25^{\circ}\left(\mathrm{CHCl}_{3} ; c 0.5\right)$, lit. [11], oil; $[\alpha]_{436 \mathrm{~nm}}^{24}-35.4^{\circ}$ $\left(\mathrm{CHCl}_{3} ; c 1.0\right) .{ }^{13} \mathrm{C} \mathrm{NMR}\left(\mathrm{CDCl}_{3}, 75 \mathrm{MHz}\right): \delta 11.6(\mathrm{C}-14)$, 21.4 (C-12), 21.7 (C-13), 22.7 (C-8), 27.0 (C-2), 29.4 (C-15), 35.1 (C-9), 35.3 (C-11), 38.1 (C-10), 38.7 (C-3), 49.8 (C-5), 71.1 (C-4), 78.4 (C-1), 115.4 (C-6), 136.0 (C-7).

Oplodiol (19). Mp 106-107,$[\alpha]_{\mathrm{D}}^{25}-52^{\circ}\left(\mathrm{CHCl}_{3} ; c 0.8\right)$, lit. [10], mp 107-108 $;[\alpha]_{\mathrm{D}}^{27}-58.0^{\circ}\left(\mathrm{CHCl}_{3} ; c 1.0\right)$.

$\beta$-Eudesmol (20). Mp 79-80, $[\alpha]_{\mathrm{D}}^{25}+56^{\circ}\left(\mathrm{CHCl}_{3}\right.$; c 1.3), lit. [13], mp 79-80; $[\alpha]_{\mathrm{D}}+56.6^{\circ}\left(\mathrm{CHCl}_{3} ; c 2.0\right)$.

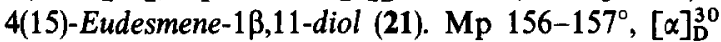
$+56^{\circ}\left(\mathrm{CHCl}_{3} ; c 1.5\right)$, lit. [14], mp 156-157 $;[\alpha]_{\mathrm{D}}^{31}$ $+56.4^{\circ}\left(\mathrm{CHCl}_{3} ; c 1.5\right)$.

4(15)-Eudesmene-1 $\beta, 6 \alpha$-diol (22). Oil, $[\alpha]_{\mathrm{D}}^{25}+7.5^{\circ}$ $\left(\mathrm{CHCl}_{3} ; c 1.2\right)$, lit. [15], gum; $[\alpha]_{436 \mathrm{~nm}}^{24}+16^{\circ}\left(\mathrm{CHCl}_{3}\right.$; c 0.1). ${ }^{13} \mathrm{C} \mathrm{NMR}\left(\mathrm{CDCl}_{3}, 75 \mathrm{MHz}\right): \delta 11.6(\mathrm{C}-14), 18.2$ (C8), 16.2 (C-12), 21.1 (C-13), 26.0 (C-11), 31.9 (C-2), 35.1 (C3), 36.3 (C-9), 41.7 (C-10), 49.3 (C-7), 55.9 (C-5), 67.0 (C-6), 79.0 (C-1), 107.8 (C-15), 146.2 (C-4).

$1 \beta$-Acetoxy-4(15)-eudesmen-11-ol (23). Oil, $[\alpha]_{\mathrm{D}}^{30}+29^{\circ}$ $\left(\mathrm{CHCl}_{3} ; c 2.2\right)$. TLC (EtOAc- $\mathrm{CHCl}_{3}$-hexane, 5:50:45) $R_{f} 0.27$. IR $v_{\max }^{\text {neat }} \mathrm{cm}^{-1}: 3453,3079,1711,888$. EIMS (70 eV) $m / 2$ (rel. int.): $202\left[\mathrm{M}-\mathrm{H}_{2} \mathrm{O}-\mathrm{HOAc}^{+}(15)\right.$, 162 (100), 147 (65), 133 (20), 119 (18), 106 (16), 59 (18). HRMS for $\mathrm{C}_{17} \mathrm{H}_{28} \mathrm{O}_{3}$ requires 280.2039 ; found 280.2021 . Saponification of $23(20 \mathrm{mg})$ by a procedure similar to that for 8 gave 21 (16 mg).

$\alpha$-Cadinol (24). Mp 70-71 ${ }^{\circ},[\alpha]_{\mathrm{D}}^{25}-45^{\circ}\left(\mathrm{CHCl}_{3} ; c 0.8\right)$, lit. $[17], \mathrm{mp} 72.5^{\circ} ;[\alpha]_{\mathrm{D}}-39.4^{\circ}\left(\mathrm{CHCl}_{3} ; c 1.32\right)$.

T-Cadinol (25). Oil, $[\alpha]_{\mathrm{D}}^{25}-5^{\circ}\left(\mathrm{CHCl}_{3} ; c 1.2\right)$, lit. [18], oil; $[\alpha]_{\mathrm{D}}^{30}-4.7^{\circ}\left(\mathrm{CHCl}_{3} ; c 4.4\right)$.

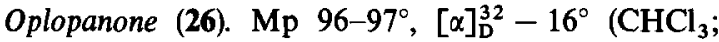
$c 2.0$ ), lit. [19], mp 96-97; $[\alpha]_{\mathrm{D}}^{25.5}-20.0^{\circ}$ (dioxane; c0.571). ${ }^{13} \mathrm{CNMR}\left(\mathrm{CDCl}_{3}, 75 \mathrm{MHz}\right): \delta 15.6$ (C-12), 20.2 (C-14), 21.9 (C-13), 23.0 (C-8), 25.3 (C-2), 28.6 (C-3), 29.4 (C-15), 29.5 (C-11), 42.0 (C-9), 46.7 (C-7), 49.4 (C-1), 55.7 (C-6), 57.0 (C-5), 73.0 (C-10), 211.4 (C-4).

Cedrol (27). Mp 80-81,$[\alpha]_{\mathrm{D}}^{25}+3^{\circ}\left(\mathrm{CHCl}_{3} ;\right.$ c 2.2), lit. $[20], \mathrm{mp} \mathrm{86}-87^{\circ} ;[\alpha]_{\mathrm{D}}^{28}+9.9^{\circ}\left(\mathrm{CHCl}_{3} ; c 5\right)$.
Acknowledgement-We thank the National Science Council for financial support (NSC83-0208-M002-095).

\section{REFERENCES}

1. Su, W.-C., Fang, J.-M. and Cheng, Y.-S. (1993) Phytochemistry 34, 779.

2. Su, W.-C., Fang, J.-M. and Cheng, Y.-S. (1994) Phytochemistry 35, 1279.

3. Su, W.-C., Fang, J.-M. and Cheng, Y.-S. (1994) Phytochemistry 37, 1109.

4. Barrero, A. F., Sanchez, J. F., Alvarez-Manzaneda, R. E. J. and Munoz Dorado, M. (1989) Phytochemistry 28, 2617.

5. Zdero, C., Bohlmann, F. and Muller, M. (1987) Phytochemistry 26, 2763.

6. Wagh, A. D., Paknikar, S. K. and Bhattacharyya, S. C. (1964) Tetrahedron 20, 2647.

7. Dolejs, L. and Herout, V. (1961) Colln Czech. Chem. Commun. 26, 2045.

8. Nanayakkara, N. P. D., Kinghorn, A. D. and Farnsworth, N. R. (1986) J. Chem. Res. (S) 454.

9. McQuillin, F. J. and Parrack, J. D. (1956) J. Chem. Soc. 2973.

10. Takahashi, K. and Takani, M. (1976) Chem. Pharm. Bull. 24, 2000.

11. Bohlmann, F., Knoll, K. H., Zdero, C., Mahanta, P. K., Grenz, M., Suwita, A. Ehlers, D., Van, N. L., Abraham, W. R. and Natu, A. A. (1977) Phytochemistry 16, 965.

12. Minato, H. and Ishikawa, M. (1967) J. Chem. Soc. C. 423.

13. Humber, D. C., Pinder, A. R. and Williams, R. A. (1967) J. Org. Chem. 32, 2335.

14. Adinarayana, D. and Syamasundar, K. V. (1982) Phytochemistry 21, 1083.

15. Bohlmann, F., Ates(Goren), N., King, R. M. and Robinsin, H. (1983) Phytochemistry 22, 1675.

16. Itokawa, H., Matsumoto, H. and Mihashi, S. (1983) Chem. Letters 1253.

17. Herout, V. and Sykora, V. (1958) Tetrahedron 4, 246.

18. Cheng, Y.-S., Kuo, Y.-H. and Lin, Y.-T. (1967) J. Chem. Soc., Chem. Commun. 565.

19. Takeda, K., Minato, H. and Ishikawa, M. (1966) Tetrahedron Suppl. 7, 219.

20. Stork, G. and Clarke Jr, F. H. (1961) J. Am. Chem. Soc. 83, 3114.

21. Fiaud, J. C., Horeau, A. and Kagan, H. B. (1977) Stereochemistry, Fundamentals and Methods, Vol. 3, p. 52. Georg Thieme Publishers, Stuttgart.

22. van Beek, T. A., Kleis, R., Posthumus, M. A. and van Veldhuizen, A. (1989) Phytochemistry 28, 1909. 